\title{
Doping quantum dimer models on the square lattice
}

\author{
Didier Poilblanc, ${ }^{1,2}$ Fabien Alet, ${ }^{1}$ Federico Becca,,${ }^{1,3}$ Arnaud Ralko, ${ }^{2}$ Fabien Trousselet, ${ }^{1}$ and Frédéric Mila ${ }^{2}$ \\ ${ }^{1}$ Laboratoire de Physique Théorique, CNRS and Université de Toulouse, F-31062 Toulouse, France \\ ${ }^{2}$ Institute of Theoretical Physics, Ecole Polytechnique Fédérale de Lausanne, BSP 720, CH-1015 Lausanne, Switzerland \\ ${ }^{3}$ CNR-INFM Democritos National Simulation Centre and International School for Advanced Studies (SISSA), I-34014 Trieste, Italy
}

(Received 9 February 2006; revised manuscript received 29 June 2006; published 26 July 2006)

\begin{abstract}
A family of models is proposed to describe the motion of holes in a fluctuating quantum dimer background on the square lattice. Following Castelnovo et al. [Ann. Phys. (N.Y.) 318, 316 (2005)], a generalized RokhsarKivelson Hamiltonian at finite doping which can be mapped on a doped interacting classical dimer model is constructed. A simple physical extension of this model is also considered. Using numerical computations and simple considerations based on the above exact mapping, we determine the phase diagram of the model showing a number of quantum phases typical of a doped Mott insulator. The two-hole correlation function generically exhibits short-range or long-range algebraic correlations in the solid (columnar) and liquid (critical) phases of the model, respectively. Evidence for an extended region of a doped valence bond solid phase exhibiting holon pairing but no phase separation is given. In contrast, we show that hole deconfinement occurs in the staggered dimer phase.
\end{abstract}

DOI: 10.1103/PhysRevB.74.014437

PACS number(s): 75.10.Jm, 75.40.Mg

Soon after the discovery of cuprate superconductors with high critical temperatures, Anderson suggested that the resonating valence bond (RVB) state is the relevant insulating parent state that becomes superconducting under (arbitrary small) hole doping. ${ }^{1}$ Such a state can alternatively be viewed as a spin liquid (SL), as it has no magnetic order and it does not break any lattice symmetry. Since then, the search for exotic SL in microscopic or effective models has been very active.

In quantum spin models, where magnetic frustration suppresses long-range magnetic order, spin liquids often compete with quantum disordered states named "valence bond solids" (VBS) which break translation symmetry. ${ }^{2}$ This is, e.g., the case in the frustrated Heisenberg model with extended-range antiferromagnetic (AF) interactions. ${ }^{3}$ In a VBS, nearest-neighbor spins pair up in bond singlets which order, e.g., along columns or in a staggered arrangement. Hole doping has also been extensively studied in Mott insulators ${ }^{4}$ and AF fluctuations have been identified as the glue for pairing. Unconventional pairing upon doping models exhibiting a VBS ground state has also been found. ${ }^{5}$

In a pioneering work Rokhsar and Kivelson introduced a quantum dimer model (QDM), a Hamiltonian acting in the space of two-dimensional fully packed dimer configurations ${ }^{6}$ (generically called $|c\rangle$ ). The dimer interaction $V$ and the dimer-flip process $J$ are schematically depicted in Fig. 1(a). As discussed in Ref. 6, the QDM can be considered as the simplest effective model to describe quantum disordered phases similar to the pseudogap phase of the cuprate superconductors. In that respect, one might think of the dimer flip term as originated directly from superexchange between copper spins. At the special point $V / J=1$, named RokhsarKivelson (RK) point, the ground state (GS) is exactly known and can be mapped onto the partition function of a classical dimer model. ${ }^{6}$ On the square lattice, the dimer-dimer correlations are algebraic, decaying as $1 / r^{2}$. This "algebraic SL" at the RK point is believed to be rather singular on the $V / J$ axis since, as shown, e.g., by numerical calculations, ${ }^{7}$ the GS is a VBS on both sides of it, a staggered phase for $V / J>1$, a columnar phase at attractive $V$ (i.e., $V<0$ ), separated from the RK point by a small region of plaquette phase. The case of nonbipartite lattices, where the RK point has a gapped GS that shows fractional excitations, ${ }^{8}$ is also of great interest. Doping was introduced in Ref. 6 and studied further later by Syljuåsen ${ }^{9}$ who computed dimer correlations and the energy of two static monomers in a background of dimers and dynamic holes. However, hole correlations of the dynamic holes themselves, have not been investigated so far.

In this paper we construct various models of doped quantum dimers with the aim to study these correlations as accurately as possible. Like the more "microscopic" $t$ - $J$ model, the lightly doped QDM also provides a realistic description of relevant quantum disordered phases (which, in fact, would be stabilized only at finite doping in the $t$ - $J$ model), while being much easier to handle numerically. ${ }^{10}$ A generalized RK Hamiltonian is introduced at finite doping. This model can be

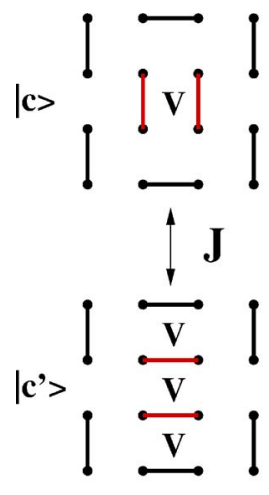

(a)

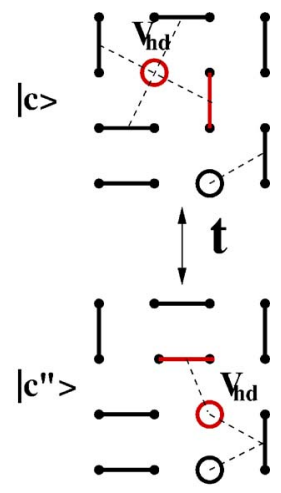

(b)
FIG. 1. (Color online) Pictures of the two quantum processes considered in this paper. (a) Dimer flip $J$ within a plaquette. A dimer repulsion $V$ is defined for all flippable plaquettes. (b) Hole hopping along a plaquette diagonal. In this process a dimer "rotates" from a vertical (horizontal) to a horizontal (vertical) bond. The hole-dimer repulsion $V_{h d}$ is defined on all the dashed lines. 


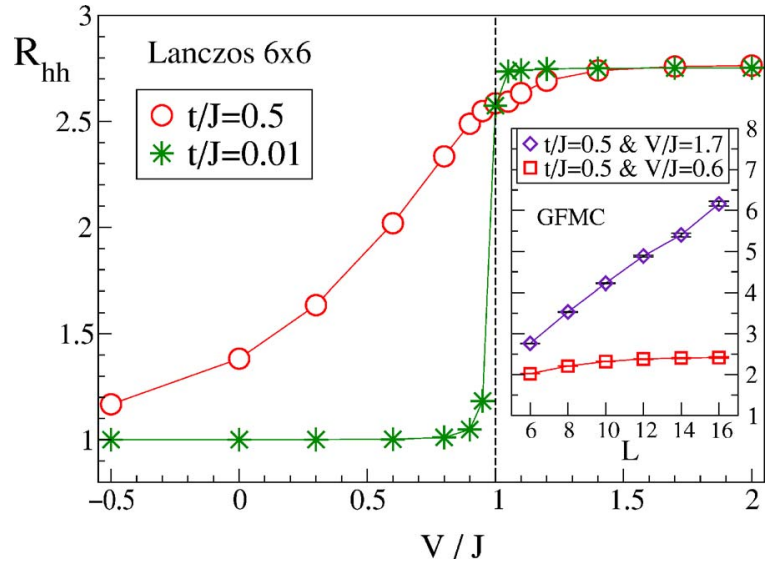

FIG. 2. (Color online) Mean-squared separation $R_{h h}$ between two holes in the $t-J-V$ model as a function of the ratio $V / J$. Calculations are done on a periodic $6 \times 6$ cluster for two values of the hopping $t / J$. Inset, size dependence of $R_{h h}$ obtained from GFMC on $L \times L$ clusters for $t / J=0.5$ and two typical values of $V / J$ showing hole-hole deconfinement and confinement, respectively.

mapped onto a doped interacting classical dimer model enabling an efficient use of classical Monte Carlo (MC). ${ }^{11}$ In addition, it offers a controllable parameter (the effective temperature) to smoothly tune the system from a VBS to a liquid phase (even at zero doping). We also extend this model to an enlarged physical space away from this so-called RK axis, where such a mapping is no longer valid ${ }^{12}$ and where full quantum computations such as Lanczos exact diagonalization (ED) and Green's function Monte Carlo (GFMC) are required. We provide evidence for hole deconfinement ${ }^{13}$ in the algebraic dimer phase. However, we also argue that phase separation occurs for low hole kinetic energy and, last, provide a complete phase diagram of the model.

Let us first assume that holes are introduced by pairs on some of the dimer bonds. Next, the simplest way to account for their motion ${ }^{6,9}$ is to consider processes like the one depicted in Fig. 1(b) where a hole hops along a plaquette diagonal with some amplitude $t$. Note that here holes are really thought of as new charge degrees of freedom originating, e.g., from doping a Mott insulator. A general form of doped QDM which operates in a Hilbert space with a fixed hole number can then be written as

$$
H=\sum_{c} \epsilon_{c}|c\rangle\left\langle c\left|-J \sum_{\left(c, c^{\prime}\right)}\right| c^{\prime}\right\rangle\left\langle c\left|-t \sum_{\left(c, c^{\prime \prime}\right)}\right| c^{\prime \prime}\right\rangle\langle c|,
$$

where the sum over $|c\rangle$ includes all configurations with arbitrary hole positions. The sum over $|c\rangle$ and $\left|c^{\prime}\right\rangle$ extends on all pairs of doped dimer coverings (only) differing by a single plaquette flip [shown in Fig. 1(a)]. Similarly, the sum over $|c\rangle$ and $\left|c^{\prime \prime}\right\rangle$ extends to all pairs of doped dimer coverings differing (only) by a single hole hopping along a diagonal of a plaquette and a single dimer "hop" from a vertical (horizontal) bond to a horizontal (vertical) bond. Such a process between two configurations $|c\rangle$ and $\left|c^{\prime \prime}\right\rangle$ is depicted in Fig. 1(b). As shown below, the role of the diagonal energies $\epsilon_{c}$ is crucial and various choices will be discussed. Whether

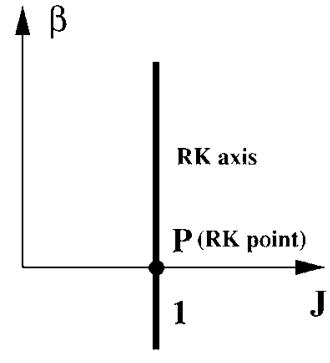

(a)

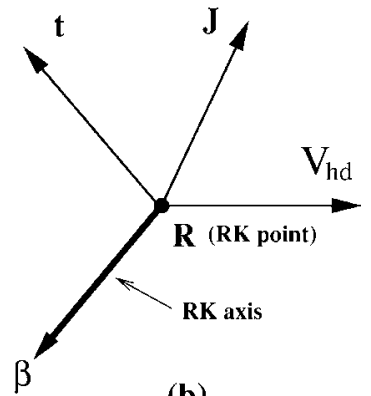

(b)
FIG. 3. Schematic picture of the parameter space of the models considered here; (a) $J$ - $\beta$ model (undoped) and (b) $t-J-\beta$ model (doped). The RK axes (thick lines parametrized by $\beta$ ) are defined by $J=1$ (undoped case) and by $J=t=V_{h d}=1$ (doped case). The points $P$ and $R$ correspond to the origins at $\beta=0$ on these axes.

mobile holes remain confined ${ }^{14}$ or not (or whether they form bound states) is the central issue of this study.

Following Ref. 6, we first start with the simple dimer interaction introduced above, namely $\epsilon_{c}=\epsilon_{c}^{0}=V N_{c}$ where $N_{c}$ corresponds to the number of flippable plaquettes in configuration $|c\rangle$. The properties of two holes ${ }^{15}$ in such a $t-J-V$ model $^{9}$ are studied here by ED and GFMC (Ref. 16) on finite clusters and the mean-squared hole-hole distance $R_{h h}=\sqrt{\left\langle r^{2}\right\rangle}$ is shown in Fig. 2 as a function of the dimer repulsion $V / J$ for fixed ratios $t / J$. A very abrupt variation is observed at $V / J=1$ (especially at small $t$ ) showing a clear confinement $^{14}$ at $V / J<1$, as expected in a VBS, and a large value of the hole-hole separation for $V / J>1$, which scales linearly with system size (see inset of Fig. 2). In this case, the undoped system is also a VBS, but a pure staggered dimer state with no quantum fluctuations. It is therefore easy to see that the two holes can freely move away from each other in opposite directions along the same diagonal creating a string of dimers at $90^{\circ}$ from the background at no energy cost. ${ }^{17}$ On the contrary, in a plaquette or columnar VBS the energy cost grows linearly with the string length leading to confinement. Note that it was argued that confinement is lost for a sufficiently high fraction of holes (monomers). ${ }^{9}$

In order to construct more general doped QDM, we briefly reexamine the undoped case [i.e., zero doping for which the $t$ term of Eq. (1) is irrelevant] and introduce a simple construction that extends the undoped RK point to an infinite axis. Following Castelnovo et al., ${ }^{18}$ we define the $J-\beta$ Hamiltonian by introducing diagonal energies as

$$
\boldsymbol{\epsilon}_{c}=\epsilon_{c}^{\mathrm{flip}}=V \sum_{c^{\prime}(c)} \exp \left(-\frac{1}{2} \beta V_{\mathrm{cl}}\left(N_{c^{\prime}}-N_{c}\right)\right),
$$

where the sum extends on the dimer covering $\left|c^{\prime}\right\rangle$ differing from $|c\rangle$ by a single plaquette flip [shown on Fig. 1(a)]. In the $\beta \rightarrow 0$ limit, the expression of $\epsilon_{c}^{\text {flip }}$ reduces to $\epsilon_{c}^{0}$ and the significance of $V$ becomes clear. Hereafter, $V=1$ sets the energy scale. $V_{\mathrm{cl}}$ corresponds to a classical dimer-dimer interaction. We restrict ourselves to the attractive case and use units for $\beta$ such that $V_{\mathrm{cl}}=-1$. A cartoon of the Hamiltonian manifold parametrized by $(J, \beta)$ is shown in Fig. 3(a). For $J=1$ it is easy to check that the GS is simply given by 
$\frac{1}{\sqrt{Z}} \sum_{c} \exp \left(-\frac{1}{2} \beta V_{\mathrm{cl}} N_{c}\right)|c\rangle$ with energy $E_{0}=0$, where the normalization factor $Z=\Sigma_{c} \exp \left(-\beta V_{\mathrm{cl}} N_{c}\right)$ can be considered as a partition function of a classical interacting dimer model. ${ }^{19}$ We have checked by ED data of a $8 \times 8$ cluster within its fully symmetric space-group irreducible representation, that the specific heat [defined as $\beta^{2}\left(\left\langle N_{c}^{2}\right\rangle-\left\langle N_{c}\right\rangle^{2}\right)$ ] is very close to the MC results obtained for a very large cluster (not shown). The model displays a Kosterlitz-Thouless (KT) transition ${ }^{20}$ at $\beta=\beta_{\mathrm{KT}} \simeq 1.536$ between a critical phase at $\beta<\beta_{\mathrm{KT}}$ (with $\beta$ varying exponents) and a columnar dimer phase. ${ }^{19}$ Independently from our investigation, a similar mapping was derived and the properties of this critical phase were investigated with transfer-matrix techniques. ${ }^{21}$

To investigate the expected confinement-deconfinement transition ${ }^{13}$ for holes at the KT transition, let us now generalize the construction by Castelnovo et al. ${ }^{18}$ to finite doping. We define the $t-J-\beta$ Hamiltonian by adding a second diagonal term to the one (2) of the $J-\beta$ Hamiltonian, $\epsilon_{c}=\epsilon_{c}^{\text {flip }}+\epsilon_{c}^{\text {hop }}$,

$$
\epsilon_{c}^{\mathrm{hop}}=V_{h d} \sum_{c^{\prime \prime}(c)} \exp \left(-\frac{1}{2} \beta V_{\mathrm{cl}}\left(N_{c^{\prime \prime}}-N_{c}\right)\right),
$$

where the sum now extends on the doped dimer coverings $\left|c^{\prime \prime}\right\rangle$ connected to $|c\rangle$ by a $t$ process [see again Fig. 1(b)]. Note that this new term, as the $t$ term of (1), scales like the hole concentration. $V_{h d}$ is a new energy scale which naturally makes sense in the $\beta \rightarrow 0$ limit discussed below. The term (3) is of central importance as for $t=V_{h d}$ and $J=1$ the GS can again be written as $\frac{1}{\sqrt{Z}} \sum_{c} \exp \left(-\frac{1}{2} \beta V_{\mathrm{cl}} N_{c}\right)|c\rangle$. In analogy with the undoped case, we can again define a RK axis which runs along an orthogonal direction to the three-dimensional parameter space spanned by $J, t$, and $V_{h d}$. This RK axis is given by $J=1, t=V_{h d}$ and parametrized by $\beta$. A cartoon picture of this set of Hamiltonians is shown in Fig. 3(b). Since the hopping term (for $t \neq 0$ ) couples all topological symmetry sectors the GS with energy $E_{0}=0$ becomes unique. Interestingly, the procedure followed here can be generalized to more complicated dimer or hole kinetic off-diagonal processes.

The $\beta \rightarrow 0$ limit is of special interest. As seen above, the diagonal term (2) reduces to a dimer-dimer repulsion of magnitude $V$ (set to 1) and the undoped RK point is recovered for $J=1$ (when holes are not present). Similarly, the second diagonal contribution (3) reduces to a dimer-hole interaction on a plaquette [with definition given pictorially in Fig. 1(b)] of magnitude $V_{h d}$. The $\beta=0$ limit therefore gives rise to a large class of physical Hamiltonians parametrized by arbitrary magnitudes of $J, t$, and $V_{h d}$ (measured in units of $V=1)$. A complete investigation of this model is left for a future study ${ }^{22}$ and, in the following, we restrict ourselves to $J=V$ (=1 for convenience) so that the "distance" from the RK axis will be controlled by the deviation of $t$ from $V_{h d}$.

We first start with the case of two holes in the $t-J-\beta$ model. The hole-hole correlations have been computed by $\mathrm{ED}$ of a $6 \times 6$ cluster for arbitrary $t$ and, for convenience, for $V_{h d}=1$. Results are shown in Fig. 4. The ED results show a rather smooth variation of $R_{h h}$ across the transition at $\beta_{\mathrm{KT}}$. However, the finite size scaling at $t=1$ obtained by classical

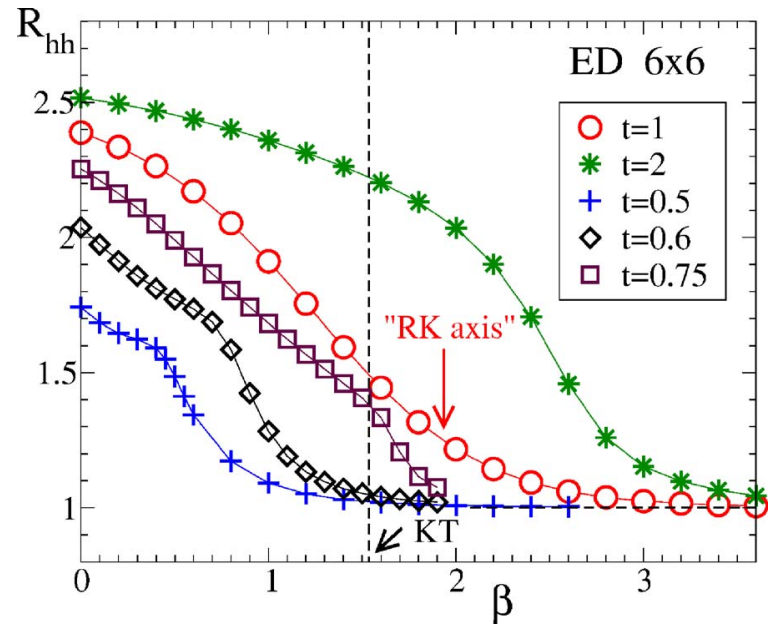

FIG. 4. (Color online) Mean-squared separation between two holes in the $t-J-\beta$ model as a function of $\beta$. ED data obtained on a $6 \times 6$ periodic cluster are shown for several values of the hopping $t$ and $J=1$. The dashed line denotes the location of the KT transition at $\beta_{\mathrm{KT}} \sim 1.536$. For $t=0.5$ and $t=0.6$ the "kinks" signal the appearance of a two-hole bound state.

MC in Fig. 5(a) shows a clear qualitative change of behavior at the KT transition: while $R_{h h}$ remains finite in the confined phase, it diverges as a power law in the critical phase in agreement with Ref. 19. For $t=0.5$ and $t=0.6$ we observe a kink in the ED data of Fig. 4. Moreover, for $t \leqslant 0.25 R_{h h}$ remains always very close to 1 , even when $\beta \rightarrow 0$ (not shown). This signals the appearance of a two-hole bound state within the critical SL phase for $\beta$ below $\beta_{\mathrm{KT}}$. This scenario is supported by the GFMC data on larger systems shown in Fig. 5(b). We have checked by GFMC that this bound state, in fact, persists up to $t=1\left(=V_{h d}\right)$. These results are summarized in a phase diagram for two holes in Fig. $5(\mathrm{c})$.
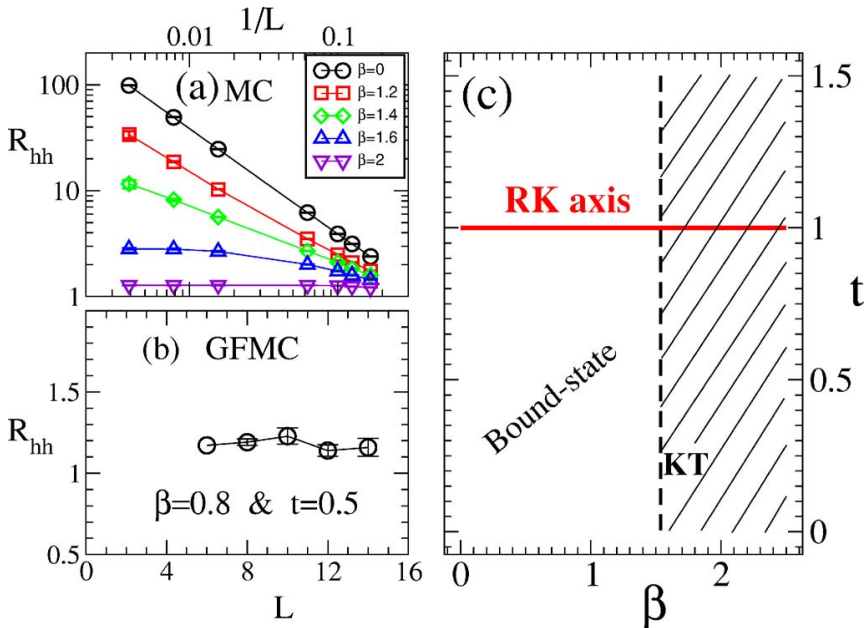

FIG. 5. (Color online) Results for two holes in the $t$ - $J$ - $\beta$ model $[J=V(=1)]$ for $V_{h d}=V(=1)$. Size dependence of $R_{h h}$ by (a) classical MC at $t=1$ for various values of $\beta$ (log-log scale), (b) GFMC at $\beta=0.8$ and $t=0.5$ (linear scale). (c) Conjectured phase diagram for two holes in the thermodynamic limit for $J=V=V_{h d}(=1)$ vs $\beta$ and $t$. The dashed region corresponds to the confined phase for $\beta>\beta_{\mathrm{KT}}$. 


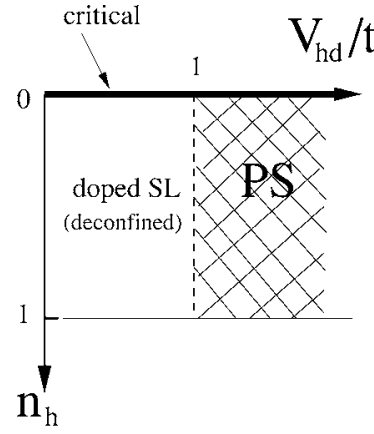

(a)

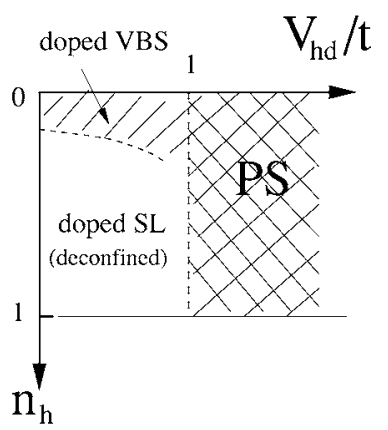

(b)
FIG. 6. Phase diagrams of the $t-J-\beta$ for $V=J$ vs hole density and $V_{h d} / t$ ratio. "PS" stands for "phase separation." (a) Phase diagram for $\beta<\beta_{\mathrm{KT}}$; (b) phase diagram for $\beta>\beta_{\mathrm{KT}}$. The boundary between the confined (doped VBS) and deconfined regions is schematic.

Next, using simple arguments, we construct the phase diagram of the doped QDM for $V=J$ (and bosonic holes). The previous finding that two holes pair up in some region of parameter space implies that either (i) a $Q=2 e$ superconducting state or (ii) a phase separated state occurs at finite doping. For $V_{h t}=t$, the inverse compressibility $\kappa^{-1}$ (proportional to the second derivative of the energy per site with respect to the hole density) identically vanishes since the GS energy vanishes on the RK axis for all number of holes. In addition, since $V_{h d}$ acts effectively as an attraction between holes (or dimers), $\kappa^{-1}$ should be a monotonous function of the ratio $V_{h d} / t$ as checked numerically. ${ }^{22}$ These simple considerations imply that $\kappa^{-1}<0(>0)$ for $t<V_{h d}\left(t>V_{h d}\right)$ and, then, the phase separation boundary is exactly given by $t=V_{h d}$ for all hole density and $\beta$. Figure 6 shows the phase diagram of the model for $J=V$ as a function of $t, V_{h d}$ and hole density. For $\beta<\beta_{\mathrm{KT}}$, holons are deconfined in the SL phase. We also expect a similar phase diagram for $\beta>\beta_{\mathrm{KT}}$ [Fig. 6(b)] although the critical line (at $n_{h}=0$ ) should be replaced by an extended (hole pair) region with VBS columnar order which survives up to a critical doping. However, the boundary of the phase separated region remains unchanged. The exact curve for the VBS/SF boundary remains to be investigated in more details.
Last, we would like to comment briefly on the case $V / J \neq 1$. In fact, we expect some qualitative similarities between the phase diagram of the "standard" (i.e., $\beta=0$ ) QDM at $V / J<1$ which exhibits a (plaquette or columnar) VBS and the low temperature VBS phase studied here at $V / J=1$. However, we believe that the phase separation line of Fig. 6(b) should be curved instead of vertical. Further investigations are needed to clarify this point. On the contrary, for $V / J>1$ (and $\beta=0$ ), in the staggered dimer phase, one would not expect any phase separation since deconfinement occurs even for two mobile holes.

In conclusion, we have introduced a class of simple doped QDM on the square lattice which, we believe, provide insights on the physical quantum disordered phases of real materials. An exact mapping onto a doped classical dimer model (characterized by an inverse temperature $\beta$ ) can be realized along a one-dimensional manifold of this multidimensional space. Using numerical computations and simple considerations based on the above exact mapping, we determine the complete phase diagram of the model (at $V=J$ ) showing a number of interesting physical phenomena that, we believe, could be generic in the vicinity of a Mott insulator, beyond the framework of QDM. It is found that two doped holons are confined in the columnar VBS phase. At finite doping, evidence for an extended region of a metallic VBS phase exhibiting holon pairing without phase separation is given. In contrast, we find that the algebraic dimer-dimer correlations of the critical (quasiordered) phase do not provide holon confinement. Furthermore, as shown in the case of the staggered dimer phase, we point out that holon deconfinement can even occur in a VBS.

The authors thank IDRIS (Orsay, France) for allocation of CPU time on the NEC-SX5 supercomputer and the Agence Nationale de la Recherche (France) for support. The authors are indebted to P. Pujol for many useful suggestions and thank G. Misguich for interesting discussions. One of the authors (D.P.) also thanks S. Kivelson for useful comments. This work was partially supported by the Swiss National Fund and by MaNEP. One of the authors (F.B.) is partially supported by COFIN 2004, COFIN 2005 (Italy), and by CNRS (France).
${ }^{1}$ P. W. Anderson, Science 235, 1196 (1987); P. W. Anderson, G. Baskaran, Z. Zou, and T. Hsu, Phys. Rev. Lett. 58, 2790 (1987).

${ }^{2}$ N. Read and S. Sachdev, Phys. Rev. Lett. 62, 1694 (1989).

${ }^{3}$ H. J. Schulz, T. Ziman, and D. Poilblanc, in Magnetic Systems with Competing Interactions, edited by H. T. Diep (World Scientific, Singapore, 1994), pp. 120-160; G. Misguich and C. Lhuillier, in Frustrated Spin Models, edited by H. T. Diep (World Scientific, New Jersey, 2004).

${ }^{4}$ E. Dagotto, Rev. Mod. Phys. 66, 763 (1994); P. A. Lee, N. Nagaosa, and X.-G. Wen, ibid. 78, 17 (2006).

${ }^{5}$ D. Poilblanc, Phys. Rev. Lett. 93, 197204 (2004).

${ }^{6}$ D. S. Rokhsar and S. A. Kivelson, Phys. Rev. Lett. 61, 2376 (1988).
${ }^{7}$ S. Sachdev, Phys. Rev. B 40, 5204 (1989); P. W. Leung, K. C. Chiu, and K. J. Runge, ibid. 54, 12938 (1996); O. F. Syljuåsen, cond-mat/0512579 (unpublished).

${ }^{8}$ R. Moessner and S. L. Sondhi, Phys. Rev. Lett. 86, 1881 (2001); G. Misguich, D. Serban, and V. Pasquier, ibid. 89, 137202 (2002); A. Ralko, M. Ferrero, F. Becca, D. Ivanov, and F. Mila, Phys. Rev. B 71, 224109 (2005).

${ }^{9}$ O. F. Syljuåsen, Phys. Rev. B 71, 020401(R) (2005); Note that in this work and in Ref. 6 an additional hopping at distance 2 is also assumed.

${ }^{10}$ Fermionic spin-1/2 spinon degrees of freedom are not included in the QDM, which is justified in pseudo-gap or spin-gap phases for energies below the gap. 
${ }^{11}$ A. W. Sandvik and R. Moessner, cond-mat/0507277 (unpublished).

${ }^{12}$ This is important as properties of RK Hamiltonians are not believed to be generic, as they essentially realize $d$-dimensional classical phases.

${ }^{13}$ We abusively use the term "deconfinement" for the critical liquid phase whereas strictly speaking, a marginal confinement still occurs due to the power-law hole-hole correlations [see W. Krauth and R. Moessner, Phys. Rev. B 67, 064503 (2003)].

${ }^{14}$ By "confinement" we implicitly mean (based on qualitative arguments) that the onset of the two hole continuum is pushed up to (strictly speaking) infinite energy. This is fundamentally different from a simple two hole bound state which can appear in a liquid dimer state.

${ }^{15}$ For two holes (which live on separate sublattices) quantum statistics does not play a role. At finite doping, the mapping discussed here also applies for bosonic holes (holons); for a discus- sion on the statistics of holons see S. Kivelson, Phys. Rev. B 39, 259 (1989).

${ }^{16}$ The GFMC technique has been used with a fixed number of walkers. For details see M. Calandra Buonaura and S. Sorella, Phys. Rev. B 57, 11446 (1998) and references therein.

${ }^{17}$ N. Shannon, G. Misguich, and K. Penc, Phys. Rev. B 69, 220403(R) (2004).

${ }^{18}$ C. Castelnovo et al., Ann. Phys. (N.Y.) 318, 316 (2005); original ideas along this line can be also found in C. L. Henley, J. Phys.: Condens. Matter 16, S891 (2004).

${ }^{19}$ F. Alet, J. L. Jacobsen, G. Misguich, V. Pasquier, F. Mila, and M. Troyer, Phys. Rev. Lett. 94, 235702 (2005).

${ }^{20}$ J. M. Kosterlitz and D. M. Thouless, J. Phys. C 6, 1181 (1973).

${ }^{21}$ C. Castelnovo, C. Chamon, C. Mudry, and P. Pujol, cond-mat/ 0602237 (unpublished).

${ }^{22} \mathrm{~F}$. Trousselet et al. (unpublished). 